\title{
Plasmon-mediated Coulomb drag between graphene waveguides
}

\author{
Shylau, Artsem A.; Jauho, Antti-Pekka
}

Published in:

Physical Review B

Link to article, DOI:

10.1103/PhysRevB.89.165421

Publication date:

2014

Document Version

Publisher's PDF, also known as Version of record

Link back to DTU Orbit

Citation (APA):

Shylau, A. A., \& Jauho, A-P. (2014). Plasmon-mediated Coulomb drag between graphene waveguides. Physical Review B, 89(16), 165421. https://doi.org/10.1103/PhysRevB.89.165421

\section{General rights}

Copyright and moral rights for the publications made accessible in the public portal are retained by the authors and/or other copyright owners and it is a condition of accessing publications that users recognise and abide by the legal requirements associated with these rights.

- Users may download and print one copy of any publication from the public portal for the purpose of private study or research.

- You may not further distribute the material or use it for any profit-making activity or commercial gain

- You may freely distribute the URL identifying the publication in the public portal

If you believe that this document breaches copyright please contact us providing details, and we will remove access to the work immediately and investigate your claim 


\title{
Plasmon-mediated Coulomb drag between graphene waveguides
}

\author{
Artsem A. Shylau* and Antti-Pekka Jauho \\ Center for Nanostructured Graphene (CNG), Department of Micro- and Nanotechnology, DTU Nanotech, \\ Technical University of Denmark, DK-2800 Kongens Lyngby, Denmark \\ (Received 10 February 2014; revised manuscript received 9 April 2014; published 24 April 2014)
}

\begin{abstract}
We analyze theoretically charge transport in Coulomb coupled graphene waveguides (GWGs). The GWGs are defined using antidot lattices, and the lateral geometry bypasses many technological challenges of earlier designs. The drag resistivity $\rho_{D}$, which is a measure of the many-particle interactions between the GWGs, is computed for a range of temperatures and waveguide separations. It is demonstrated that for $T>0.1 T_{F}$ the drag is significantly enhanced due to plasmons, and that in the low-temperature regime a complicated behavior may occur. In the weak coupling regime the dependence of drag on the interwaveguide separation $d$ follows $\rho_{D} \sim d^{-n}$, where $n \simeq 6$.
\end{abstract}

DOI: 10.1103/PhysRevB.89.165421

PACS number(s): 72.80.Vp, 73.20.Mf, 72.15.Nj, 81.05.ue

\section{INTRODUCTION}

An electric current in one conductor can induce a voltage in a neighboring conductor even though the two systems are electrically isolated. This phenomenon-Coulomb drag-has a rich phenomenology and it has been studied extensively in coupled quantum wells since the pioneering experiments by Gramila et al. [1]. Coulomb drag is a unique transport phenomenon in the sense that the signal is entirely determined by the Coulomb interaction, and thus it provides detailed insight into the many-particle interactions in low-dimensional systems. Two recent developments have further enhanced the importance of Coulomb drag. On one hand, samples with graphene layers separated by a nanometer thick boron nitride insulator enter into a new parameter regime, where the interlayer distance is shorter than the mean carrier separation in the two layers [2,3]. On the other hand, new technologies in sample preparation have allowed the study of drag between one-dimensional (1D) quantum wires, which is particularly interesting because of the expected Luttinger liquid formation [4-6], thus making the plethora of existing theoretical predictions accessible to experimental tests (e.g., Refs. [7-12]).

In the present paper we introduce and analyze a device concept which allows one to study Coulomb drag in onedimensional graphene systems (see Fig. 1) in a technologically favorable geometry. The device consists of two graphene waveguides (GWGs), defined with the help of graphene antidot lattices (GALs). The lateral geometry makes an independent contacting of the two waveguides relatively simple, it avoids complicated gatings [5], and no difficult vertical integration is required as in stacked geometries [6]. Also the graphene waveguide geometry allows one to avoid complications associated with different electronic properties depending on the orientation of the graphene lattice (zigzag or armchair). The boundary conditions utilized in the Dirac model for GAL defined waveguides do not involve the precise atomistic structure of the edges and thus make a unified description possible. The device design is based on the following considerations. The antidot lattice creates a band gap [13], which can theoretically reach several hundreds of meV [14], and thus effectively separates the two waveguides. Theoretical estimates show that three to five rows of antidots provide sufficient electrical isolation [15], implying a minimal separation of a few tens of nanometers. The waveguides defined via GALs have been shown to have good conduction properties [16], i.e., they are not so severely affected by disorder as graphene nanoribbons fabricated via an etching process [4]. A number of experimental techniques are available for the fabrication of GALs, including block-copolymer [17] and nanosphere [18] masks, ion beam etching [19], and $e$-beam lithography [20].

The proposed device geometry is, in addition to studies of Coulomb drag, highly relevant to other studies of coupled one-dimensional (1D) structures based on graphene. For example, the propagation of plasmons [21,22], or the effect of a van der Waals interaction [23], have been investigated recently both theoretically and experimentally in similar systems.

The paper is organized as follows. In Sec. II we describe three basic ingredients entering our calculations: the model for GAL waveguides, the Coulomb drag theory, and the evaluation of the dielectric function in the random phase approximation (RPA). Section III presents our numerical results and conclusions, which are summarized in Sec. IV.

\section{MODEL}

\section{A. Graphene antidot lattice waveguide}

Low energy excitations in graphene waveguides can be modeled by the Dirac equation with a mass term $m(y)$, which describes the region of graphene sheet with antidots [16], i.e., $m(y)>0,|y|>W / 2$, where $W$ is a width of the waveguide [see Fig. 1(a)]. Thus we have to solve the Schrödinger equation $\hat{H} \psi(\mathbf{r})=E \psi(\mathbf{r})$, with the Hamiltonian

\footnotetext{
*arts@nanotech.dtu.dk
}

$$
\hat{H}=\hbar v_{F}\left(\begin{array}{cc}
m(y) & -i \frac{\partial}{\partial x}-\frac{\partial}{\partial y} \\
-i \frac{\partial}{\partial x}+\frac{\partial}{\partial y} & -m(y)
\end{array}\right) .
$$


(a)
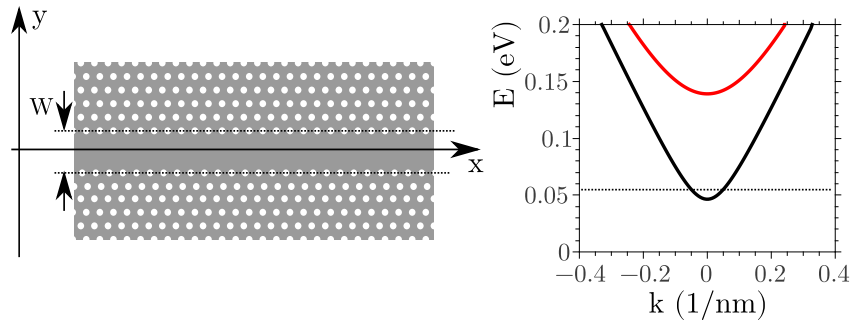

(c)

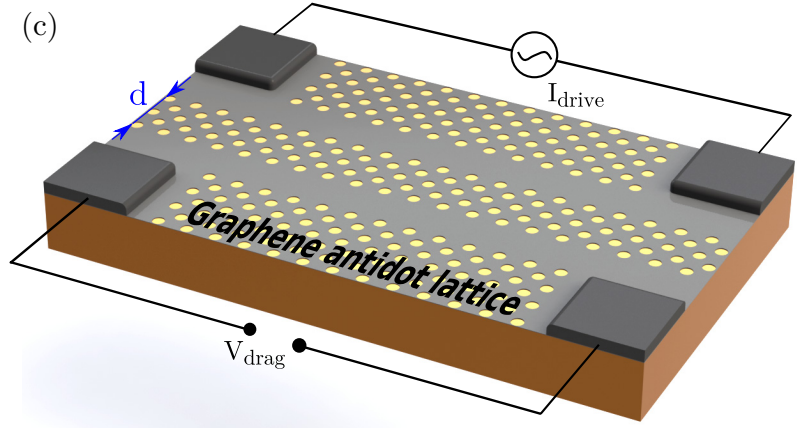

FIG. 1. (Color online) (a) Schematic illustration of a graphene waveguide $(\mathrm{GWG})$ : a region of pristine graphene of width $W$ sandwiched between regions of GALs. (b) Dispersion relation of GWG with $W=20 \mathrm{~nm}$; the black dotted line shows a representative Fermi level $E_{F}=0.054 \mathrm{eV}$, which corresponds to the charge density $n \simeq 3 \times 10^{11} \mathrm{~cm}^{-2}$. (c) Coulomb drag setup: two parallel GWGs separated by the region of GAL of the width $d$.

Due to the translational symmetry in the $x$ direction, the solution of Eq. (1) can be written in the form

$$
\psi_{n}(\mathbf{r})=e^{i k x}\left(\begin{array}{l}
\phi_{a}(y) \\
\phi_{b}(y)
\end{array}\right) .
$$

We assume that the bang gap produced by GALs is much larger than the Fermi energy, i.e., $E_{g}^{\mathrm{GAL}} \gg E_{F}$, which is mathematically expressed as $m \rightarrow \infty,|y|>W / 2$ (the infinite mass limit). Then, by applying the Berry-Mondragon boundary conditions [24], we get the wave function

$$
\psi_{n}(\mathbf{r})=\frac{1}{2 \sqrt{W} \sqrt{L}} e^{i k x}\left(\begin{array}{l}
s e^{i \theta} e^{-i k_{n} y}+e^{i k_{n} y} \\
s e^{i \theta} e^{i k_{n} y}+e^{-i k_{n} y}
\end{array}\right),
$$

where $\theta_{k_{n}, k}=\arctan \left(k_{n} / k\right)$ and $s=\operatorname{sgn}(E)$. The energy dispersion is given by a set of subbands

$$
E_{n}(k)=s \hbar v_{F} \sqrt{k^{2}+k_{n}^{2}}, \quad k_{n}=\frac{\pi}{W}\left(n+\frac{1}{2}\right) .
$$

The lowest energy excitations can be approximated by quadratic dispersion

$$
E_{k} \equiv E_{0}(k)=\hbar v_{F} \sqrt{k_{0}^{2}+k^{2}} \approx \frac{E_{g}}{2}+\frac{\hbar^{2} k^{2}}{2 m^{\star}},
$$

with the effective mass $m^{\star}=k_{0} \hbar / v_{F}$ and the band gap $E_{g}=2 \hbar v_{F} k_{0}$. If the Fermi energy lies in the lowest subband, the density of carriers is $n=\frac{g_{s} g_{v}}{\pi W} k_{F}$, where $g_{s}=g_{v}=2$ is a spin and valley degeneracy and $k_{F}$ is a Fermi wave vector. We emphasize that even though the appropriate dispersion is parabolic, the pseudospin nature of graphene permeates in the calculations due to the wave-function overlap factor discussed below.

\section{B. Drag calculation}

We use the standard expression for the drag resistivity, where the subsystem interaction is taken into account perturbatively up to second order [25-27],

$$
\begin{aligned}
\rho_{21}= & \frac{\hbar^{2}}{16 \pi e_{1} e_{2} n_{1} n_{2} k_{B} T} \frac{1}{W} \int_{-\infty}^{\infty} \frac{d q}{2 \pi} \int_{-\infty}^{\infty} d \omega\left|U_{12}(q, \omega)\right|^{2} \\
& \times \frac{\Gamma_{1}(q, \omega) \Gamma_{2}(q, \omega)}{\sinh ^{2}\left(\frac{\hbar \omega}{2 k_{B} T}\right)}
\end{aligned}
$$

where the subscript $i=1,2$ defines the waveguide and $T$ is the temperature, $\Gamma_{i}(q, \omega)$ is the nonlinear susceptibility, and $U_{12}(q, \omega)$ is the Fourier component of the screened interwaveguide Coulomb interaction.

In what follows we consider only the lowest subband. This approximation can be justified by the following arguments. First, for the parameters chosen for the calculations, namely, $E_{g} \gg k_{B} T$, the contribution from the interband transition is small compared to the intraband contribution. Also, an analysis of Eq. (6) shows that the drag resistivity decreases rapidly within increasing Fermi level. If the charge densities are equal in both waveguides, the drag resistivity scales by the factor $1 / n^{2}$. Moreover, as we show in detail below, the dominant contribution to $\rho_{21}$ comes from backscattering with momentum transfer $k_{F} \lesssim q \lesssim 2 k_{F}$ (see Fig. 3). In this case the interaction between waveguides, described by the $U_{12}(q, \omega)$ term, decays rapidly with an increase of $E_{F}$. Therefore, in order to get a measurable signal, one has to operate at low doping, which corresponds to the Fermi level located in the vicinity of the lowest subband edge.

The nonlinear susceptibility, which describes a response of the charge density to an external potential, is given in the Boltzmann limit (weak disorder) by [28]

$$
\begin{aligned}
\Gamma_{i}(q, \omega)= & \frac{2 \pi e_{i} g_{s} g_{v}}{\hbar \mu_{\mathrm{tr}, i}} \int_{-\frac{\pi}{a}}^{\frac{\pi}{a}} \frac{d k}{2 \pi} \delta\left(E_{k}-E_{k+q}-\hbar \omega\right) \\
& \times\left[f\left(E_{k}\right)-f\left(E_{k+q}\right)\right]\left[\tau_{k+q} v_{k+q}-\tau_{k} v_{k}\right] \\
& \times F(k, k+q),
\end{aligned}
$$

where $\tau_{k}$ is the transport scattering time, $\mu_{\mathrm{tr}, i}$ is a mobility in a sense that $j=e n \mu_{\mathrm{tr}} E, v_{k}=\frac{1}{\hbar} \frac{\partial E(k)}{\partial k}$ is a group velocity, and $f(E)$ is the Fermi-Dirac distribution function. The function $F(k, k+q)=\left[1+\cos \left(\theta_{k_{0}, k+q}-\theta_{k_{0}, k}\right)\right] / 2$ is the wave-function overlap, which stems from the calculation of the Coulomb interaction matrix element $\left\langle k, k+q\left|V\left(\mathbf{r}_{1}, \mathbf{r}_{2}\right)\right| k+\right.$ $q, k\rangle$.

In general, the transport scattering time is a function of momentum (or energy) $\tau=\tau(\mathbf{k})$. In the low-temperature limit, $T \ll T_{F}$, which we consider here, the drag resistivity is not sensitive to the precise functional dependence of $\tau(\mathbf{k})$ [29], so that the relaxation time approximation $\tau(\mathbf{k}) \simeq \tau_{F}=$ const can be employed [30,31]. Due to the delta function in Eq. (7), the 
integral can be evaluated analytically,

$\Gamma(q, \omega)=\operatorname{sgn}(q) \frac{k_{0} g_{s} g_{v}}{\hbar v_{F}}\left[f\left(E_{k_{s}}\right)-f\left(E_{k_{s}+q}\right)\right] F\left(k_{s}, k_{s}+q\right)$,

where we used that $\mu_{\mathrm{tr}}=e \tau / m^{\star}$ and

$$
k_{s}=-\frac{k_{0}}{q} \frac{\omega}{v_{F}}-\frac{1}{2} q
$$

is a root of the equation $E_{k_{s}}-E_{k_{s}+q}-\hbar \omega=0$.

\section{Screening}

The dynamically screened interwaveguide Coulomb interaction is

$$
U_{12}(q, \omega)=\frac{V_{12}(q)}{\epsilon(q, \omega)},
$$

where $\epsilon(q, \omega)$ is the dielectric function calculated within the random phase approximation [25-27],

$$
\begin{aligned}
\epsilon(q, \omega)= & {\left[1-V_{11}(q) \Pi_{11}(q, \omega)\right]\left[1-V_{22}(q) \Pi_{22}(q, \omega)\right] } \\
& -V_{21}(q) \Pi_{11}(q, \omega) V_{12}(q) \Pi_{22}(q, \omega),
\end{aligned}
$$

and $V_{i j}(q)$ are the 1D Fourier components of the bare Coulomb interaction,

$$
V_{i j}(q)=\frac{e_{i} e_{j}}{2 \pi \epsilon_{r} \epsilon_{0}} \int \frac{d y_{1}}{W_{i}} \int \frac{d y_{2}}{W_{j}} K_{0}\left(q\left|y_{1}-y_{2}\right|\right),
$$

where $K_{0}(y)$ is a zero-order modified Bessel function, and $\epsilon_{r}=2.5$ is the relative dielectric permittivity. The finite- $T$ polarizability is given by the bare bubble diagram [32]

$\Pi_{m n}(q, \omega)=\frac{g_{s} g_{v}}{L} \sum_{k} \frac{f\left(E_{k+q}^{m}\right)-f\left(E_{k}^{n}\right)}{E_{k+q}^{m}-E_{k}^{n}-\hbar(\omega+i \eta)} F(k, k+q)$,

where $L$ is a length of the waveguides.

\section{RESULTS}

For the sake of simplicity we consider two equal GWGs, i.e., $W_{1}=W_{2}=20 \mathrm{~nm}$, with equal chemical potential and temperature [see Fig. 1(c)]. The distance between GWGs is $40 \mathrm{~nm}$. The band gap inside the waveguide, caused by quantum confinement, is $E_{g}=0.092 \mathrm{eV}$. In order to appreciate the role of screening, we first calculate the drag using a bare Coulomb interaction, i.e., $\epsilon(q, \omega)=1$. Figure 2 shows the drag resistivity as a function of temperature for different values of chemical potential. One can see that the value of $\rho_{D}$ is very sensitive to the value of $E_{F}$. An increase of $E_{F}$ by just a few meV's results in a significant drop of the drag resistivity for two reasons. First, a change of the chemical potential induces extra carries in the system, which decreases the drag resistivity because of the factor $n^{-2}$ (for equivalent waveguides) according to Eq. (6). The second reason is related to the fact that scattering with momentum transfer of the order of $k_{F}$, described by $U_{21}(q)$, is much smaller for a larger $E_{F}$.

In the case of an unscreened Coulomb interaction the temperature dependence of the drag resistivity exhibits the following behavior: At small temperatures the drag grows rapidly with increasing $T$, reaching the maximum value at
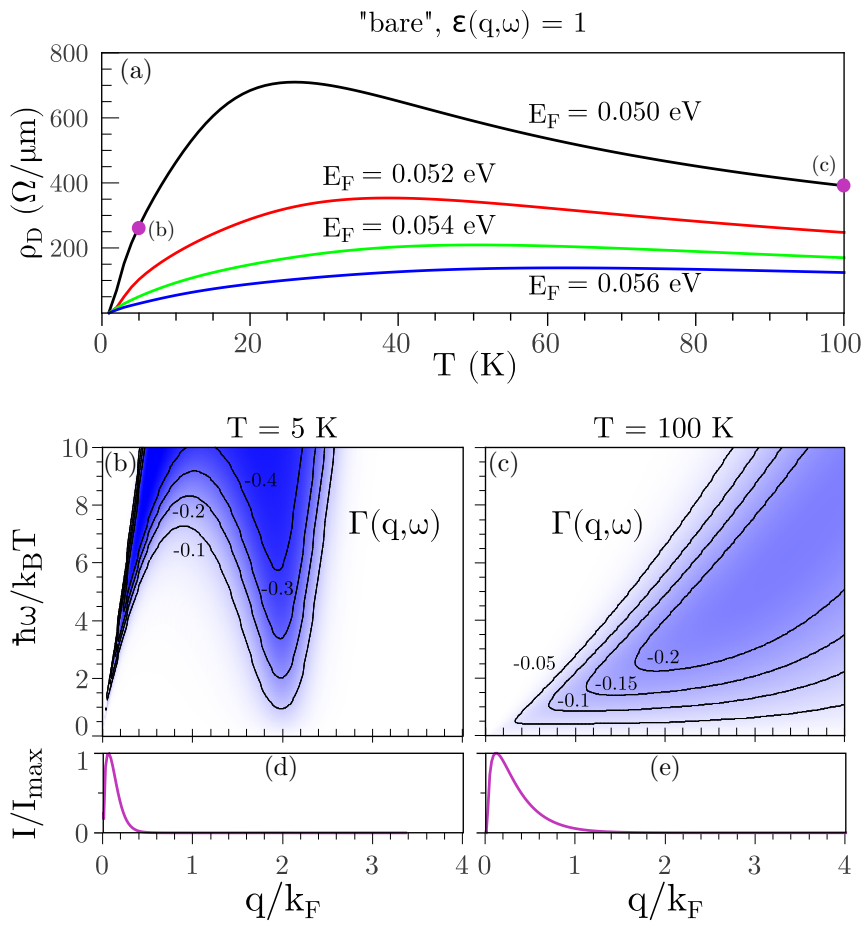

FIG. 2. (Color online) (a) Drag resistivity between equal ballistic waveguides as a function of temperature for different chemical potentials. The width of the waveguides $W_{1}=W_{2}=20 \mathrm{~nm}$ and $d=40 \mathrm{~nm}$. Nonlinear susceptibility at different (b) $T=5 \mathrm{~K}$ and (c) $T=100 \mathrm{~K}$ temperatures of the system and $E_{F}=0.050 \mathrm{eV}$. (d), (e) Normalized drag intensity calculated using Eq. (14).

$T \approx 0.05 T_{F}$. A further increase of the temperature results in either decay of $\rho_{D}$ (for $E_{F}=0.050 \mathrm{eV}$ ) or saturation of its value (for $E_{F}=0.056 \mathrm{eV}$ ). The explanation for this behavior is based on a phase-space consideration of $\Gamma(q, \omega)$ function, as we now discuss.

Figures 2(b) and 2(c) show the nonlinear susceptibility $\Gamma(q, \omega)$ as a function of transferred momentum and energy. At low temperatures $[T=5 \mathrm{~K}$, Fig. 2(b)] there are two types of excitations available: (i) forward scattering with a small momentum $q \rightarrow 0$ and (ii) backscattering with momentum transfer $q \simeq 2 k_{F}$. [Note that scattering with a momentum around $q=k_{F}$ requires a large energy transfer and is therefore suppressed due to the factor $\sinh ^{-2}\left(\hbar \omega / 2 k_{B} T\right)$ in Eq. (6).] Even though there is much more phase space available around $q=2 k_{F}$, the forward scattering with a small momentum transfer produces a dominant contribution to the drag, which can be shown by calculating the drag intensity,

$$
I(q)=\int_{-\infty}^{\infty} d \omega \frac{\left|U_{12}(q, \omega)\right|^{2} \Gamma_{1}(q, \omega) \Gamma_{2}(q, \omega)}{\sinh ^{2}\left(\hbar \omega / 2 k_{B} T\right)},
$$

as depicted in Fig. 2(d). With an increase of the temperature [ $T=100 \mathrm{~K}$, Fig. 2(c)] the phase space in between $q=0$ and $q=2 k_{F}$ is distributed almost evenly for low energy excitations. Taking into account that the matrix element of the bare Coulomb interaction grows rapidly with $q \rightarrow 0$, the forward scattering is also a dominant process at high temperatures, as shown in Fig. 2(e). 
Next we analyze the effect of screening on the drag resistivity. For this purpose we compare the drag resistivity calculated with the bare and the screened Coulomb interaction [see Fig. 3(a)]. The increase of "screened" $\rho_{D}$ with increasing temperature can be understood by using the same phase-space arguments as in the case of the drag calculated with a bare Coulomb interaction. However, at small temperatures, $\rho_{D}^{\text {screened }} \ll \rho_{D}^{\text {bare }}$. This is an intuitively expected result, since screening normally lowers the interaction and hence suppresses the drag. With a further increase of the temperature the relation between the drag calculated with a bare and screened interaction becomes opposite, $\rho_{D}^{\text {screened }} \gg \rho_{D}^{\text {bare }}$. We attribute this behavior to a plasmon-mediated enhancement of the Coulomb drag. Since the drag depends on the screened Coulomb interaction $U_{12}(q, \omega)=V_{12}(q, \omega) / \epsilon(q, \omega)$, for a certain $\omega(q)$ corresponding to a plasmon mode, the dielectric function tends to zero, $\operatorname{Re}[\epsilon(q, \omega)] \rightarrow 0$, which results in a large $U_{12}(q, \omega)$ and, in turn, increases the drag. Plasmon
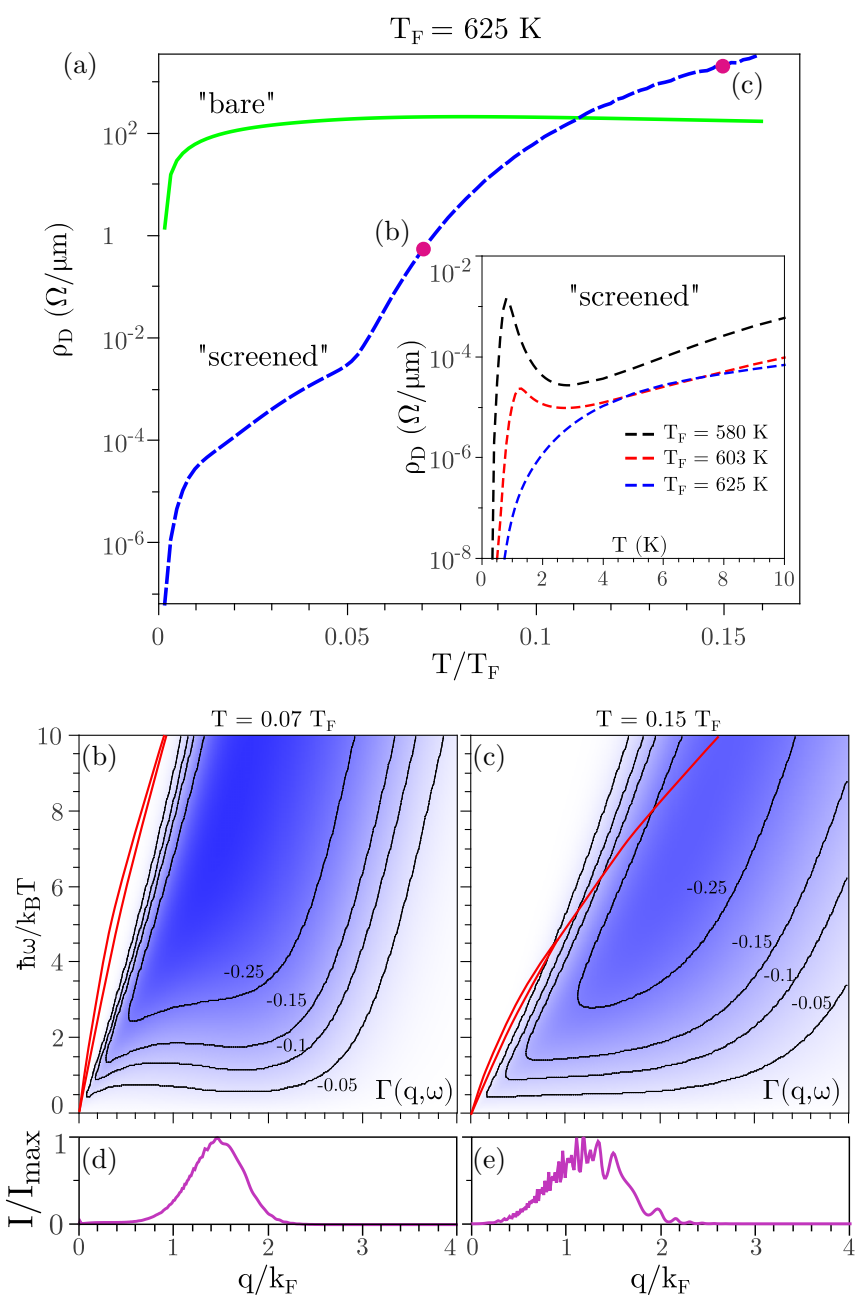

FIG. 3. (Color online) Drag resistivity as a function of temperature with unscreened (green solid line, the same as in Fig. 2) and screened (blue dashed line) Coulomb interaction. Pink dots on the curve correspond to the temperature points examined on (b) and (c). Inset: Low-temperature behavior of $\rho_{D}$. (b), (c) The nonlinear susceptibility calculated at different temperatures. Red curves show dispersion $\omega(q)$ of the plasmon modes. (d), (e) The normalized drag intensity. enhancement of the drag has been considered for coupled quantum wells in Refs. [33-35] and for two-dimensional (2D) graphene in Ref. [36]. Thus, we need the plasmon dispersion for the coupled graphene waveguides, i.e., the solutions of $\operatorname{Re}[\epsilon(q, \omega)]=0$. As it is shown in Figs. 3(b) and 3(c) (red solid lines), two plasmon modes are supported: the out-of-phase (acoustic) $\omega^{-}(q)$ and in-phase (optic) $\omega^{+}(q)$ plasmon modes. At small $q$ the modes are energy resolved and $\omega^{+}(q)>\omega^{-}(q)$, while at large $q$ the two branches merge. These coupled plasmon modes are similar to those calculated for the case of two graphene nanoribbons [22,37]. As it is shown in Fig. 3(b), at the temperature $T=0.07 T_{F}$ the plasmon modes lie outside the particle-hole continuum defined by $\Gamma(q, \omega)$. In this case the screening is effective and therefore $\rho_{D}^{\text {screened }} \ll$ $\rho_{D}^{\text {bare }}$. With an increase of the temperature $\left[T=0.15 T_{F}\right.$, Fig. 3(c)] the nonlinear susceptibility $\Gamma(q, \omega)$ is nonzero at $\omega(q)$ 's corresponding to the plasmon modes. In this case the Coulomb interaction $U_{12}(q, \omega)$ increases ("antiscreening"), which eventually leads to the enhancement of the drag. Interestingly, screening modifies also the drag intensity. Its maximum lies in between $q=k_{F}$ and $q=2 k_{F}$, as shown in Figs. 3(d) and 3(e), which means that the backscattering is a dominant process contributing to the drag.

Finally, the inset of Fig. 3(a) shows that the drag resistivity may show an upturn at the very lowest temperatures, depending sensitively on the Fermi energy. We have not identified a simple physical reason for this behavior: It is a result of a complex interplay between the various factors in the drag formula, Eq. (6). A similar behavior is predicted in drag between Luttinger liquids [38] and has been recently measured experimentally [6]. In Ref. [39] the upturn of the drag resistivity was also observed in GaAs-AlGaAs electron-hole bilayers. This effect was considered as a signature of exciton superfluidity. Intriguingly, according to their measurements, the upturn may be followed by a downturn of the drag as

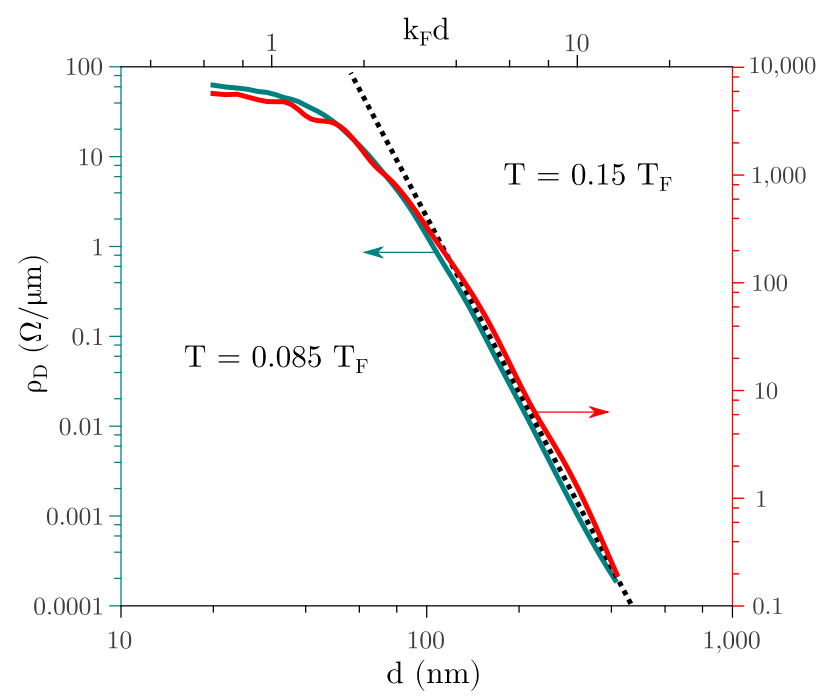

FIG. 4. (Color online) Drag resistivity between two identical $\left(W_{1}=W_{2}=20 \mathrm{~nm}\right) \mathrm{GWG}$ as a function of distance $d$ between them calculated at $T=0.085 T_{F}$ (green line, left axis) and $T=0.15 T_{F}$ (red line, right axis). The Fermi temperature is $T_{F}=580 \mathrm{~K}$ and $k_{F}=0.03181 / \mathrm{nm}$. The black dotted line illustrates asymptotic behavior in the regime $k_{F} d>1$. 
$T \rightarrow 0$. Our calculations exhibit similar trends, but arise here from single particle excitations within the Fermi liquid theory.

Finally, we investigate the interwaveguide distance dependence of the drag, which is depicted in Fig. 4. These calculations have been carried out at two representative temperatures, $T=0.085 T_{F}$ and $T=0.15 T_{F}$, which correspond to the screened and enhanced Coulomb interaction, respectively. Both curves being properly scaled have approximately the same functional dependence. However, in contrast to the case of 2D graphene sheets, where theoretical predictions $[40,41]$ and experimental measurements [2] show a $\rho_{D} \sim d^{-4}$ dependence, we find that in the weak coupling regime $k_{F} d>1$ the distance dependence of the drag between two 1D graphene wires follows $\rho_{D} \sim d^{-n}$, where $n=6.0 \pm 0.5$.

\section{SUMMARY}

In the present paper we have studied the Coulomb drag between graphene waveguides, defined with the help of a graphene antidot lattice. The energy dispersion of GWGs was calculated using the Dirac model with the effective mass term. Using the lowest-subband approximation we computed the drag resistivity. We showed that despite the relatively large interwaveguide separations required for isolated GWGs, the magnitude of Coulomb drag resistivity is in the experimentally measurable range. By performing a detailed analysis of the RPA screening, we found that plasmons provide a significant enhancement of the drag at temperatures $T>0.1 T_{F}$. At low temperatures the drag resistivity may exhibit a complicated behavior, namely, the upturn of the drag, which is always followed by downturn. Finally we showed that in the weak coupling regime the dependence of the drag on interwaveguide separation has $\rho_{D} \sim d^{-n}$ asymptotic with $n \simeq 6$. We believe that the device concept suggested here is quite versatile, and may function as a platform for many other investigations.

\section{ACKNOWLEDGMENTS}

We thank S. Badalyan for insightful remarks. The Centerof-Excellence CNG is sponsored by the Danish National Research Foundation, Project No. DNRF58.
[1] T. J. Gramila, J. P. Eisenstein, A. H. MacDonald, L. N. Pfeiffer, and K. W. West, Phys. Rev. Lett. 66, 1216 (1991).

[2] R. V. Gorbachev, A. K. Geim, M. I. Katsnelson, K. S. Novoselov, T. Tudorovskiy, I. V. Grigorieva, A. H. MacDonald, K. Watanabe, T. Taniguchi, and L. A. Ponomarenko, Nat. Phys. 8, 896 (2012).

[3] M. Titov, R. V. Gorbachev, B. N. Narozhny, T. Tudorovskiy, M. Schütt, P. M. Ostrovsky, I. V. Gornyi, A. D. Mirlin, M. I. Katsnelson, K. S. Novoselov, A. K. Geim, and L. A. Ponomarenko, Phys. Rev. Lett. 111, 166601 (2013).

[4] H. Chen and J. Appenzeller, Nano Res. 6, 897 (2013).

[5] P. Debray, V. Zverev, O. Raichev, R. Klesse, P. Vasilopoulos, and R. S. Newrock, J. Phys.: Condens. Matter 13, 3389 (2001).

[6] D. Laroche, G. Gervais, M. P. Lilly, and J. L. Reno, Science 343, 631 (2014)

[7] K. Flensberg, Phys. Rev. Lett. 81, 184 (1998).

[8] Y. V. Nazarov and D. V. Averin, Phys. Rev. Lett. 81, 653 (1998).

[9] V. V. Ponomarenko and D. V. Averin, Phys. Rev. Lett. 85, 4928 (2000).

[10] N. A. Mortensen, K. Flensberg, and A.-P. Jauho, Phys. Rev. Lett. 86, 1841 (2001).

[11] N. A. Mortensen, K. Flensberg, and A.-P. Jauho, Phys. Rev. B 65, 085317 (2002).

[12] B. Trauzettel, R. Egger, and H. Grabert, Phys. Rev. Lett. 88, 116401 (2002).

[13] T. G. Pedersen, C. Flindt, J. Pedersen, N. A. Mortensen, A.-P. Jauho, and K. Pedersen, Phys. Rev. Lett. 100, 136804 (2008).

[14] J. A. Fürst, J. G. Pedersen, C. Flindt, N. A. Mortensen, M. Brandbyge, T. G. Pedersen, and A-P. Jauho, New J. Phys. 11, 095020 (2009).

[15] T. Gunst, T. Markussen, A.-P. Jauho, and M. Brandbyge, Phys. Rev. B 84, 155449 (2011).

[16] J. G. Pedersen, T. Gunst, T. Markussen, and T. G. Pedersen, Phys. Rev. B 86, 245410 (2012).
[17] J. W. Bai, X. Zhong, S. Jiang, Y. Huang, and X. F. Duan, Nat. Nanotechnol. 5, 190 (2010).

[18] M. Wang, L. Fu, L. Gan, C. Zhang, M. Rümmeli, A. Bachmatiuk, K. Huang, Y. Fang, and Z. Liu, Sci. Rep. 3, 1238 (2013).

[19] J. Eroms and D. Weiss, New J. Phys. 11, 095021 (2009).

[20] M. Begliarbekov, O. Sul, J. Santanello, N. Ai, X. Zhang, E. H. Yang, and S. Strauf, Nano Lett. 11, 1254 (2011).

[21] A. Yu. Nikitin, F. Guinea, F. J. Garcia-Vidal, and L. MartinMoreno, Phys. Rev. B 85, 081405(R) (2012).

[22] J. Christensen, A. Manjavacas, S. Thongrattanasiri, F. H. L. Koppens, and F. Javier Garcia de Abajo, ACS Nano 6, 431 (2012).

[23] D. Drosdoff and L. M. Woods, Phys. Rev. Lett. 112, 025501 (2014).

[24] M. V. Berry and R. J. Mondragon, Proc. R. Soc. London, Ser. A 412, 53 (1987).

[25] A. P. Jauho and H. Smith, Phys. Rev. B 47, 4420 (1993).

[26] K. Flensberg, Ben Yu-Kuang Hu, A.-P. Jauho, and J. M. Kinaret, Phys. Rev. B 52, 14761 (1995).

[27] A. Kamenev and Y. Oreg, Phys. Rev. B 52, 7516 (1995).

[28] A. M. Lunde, K. Flensberg, and A.-P. Jauho, Phys. Rev. B 71, 125408 (2005).

[29] M. Carrega, T. Tudorovskiy, A. Principi, M. I. Katsnelson, and M. Polini, New J. Phys. 14, 063033 (2012).

[30] B. N. Narozhny, M. Titov, I. V. Gornyi, and P. M. Ostrovsky, Phys. Rev. B 85, 195421 (2012).

[31] B. Amorim and N. M. R. Peres, J. Phys.: Condens. Matter 24, 335602 (2012).

[32] L. Brey and H. A. Fertig, Phys. Rev. B 75, 125434 (2007).

[33] K. Flensberg and B. Y.-K. Hu, Phys. Rev. Lett. 73, 3572 (1994).

[34] N. P. R. Hill, J. T. Nicholls, E. H. Linfield, M. Pepper, D. A. Ritchie, G. A. C. Jones, B. Y.-K. Hu, and K. Flensberg, Phys. Rev. Lett. 78, 2204 (1997).

[35] K. Flensberg and B. Y.-K. Hu, Phys. Rev. B 52, 14796 (1995). 
[36] S. M. Badalyan and F. M. Peeters, Phys. Rev. B 86, 121405(R) (2012).

[37] C. E. P. Villegas, M. R. S. Tavares, G.-Q. Hai, and P. Vasilopoulos, Phys. Rev. B 88, 165426 (2013).

[38] M. Pustilnik, E. G. Mishchenko, L. I. Glazman, and A. V. Andreev, Phys. Rev. Lett. 91, 126805 (2003).
[39] A. F. Croxall, K. Das Gupta, C. A. Nicoll, M. Thangaraj, H. E. Beere, I. Farrer, D. A. Ritchie, and M. Pepper, Phys. Rev. Lett. 101, 246801 (2008).

[40] M. I. Katsnelson, Phys. Rev. B 84, 041407(R) (2011).

[41] W.-K. Tse, B. Y.-K. Hu, and S. Das Sarma, Phys. Rev. B 76, 081401(R) (2007). 